\title{
Preventing hypotension-induced nausea and vomiting during spinal anesthesia for Cesarean delivery in obese parturients: a small solution for a big problem?
}

\author{
Warwick D. Ngan Kee, BHB, MBChB, MD, FANZCA, FHKCA, FHKAM (Anaesthesiology) (1)
}

Received: 15 September 2017/ Accepted: 23 November 2017/Published online: 5 December 2017

(C) Canadian Anesthesiologists' Society 2017

Numerous studies have been published to address the problem of hypotension during spinal anesthesia for Cesarean delivery. Nevertheless, consensus on best management is lacking, and many researchers remain active in this area. In particular, the choice and use of vasopressors is especially controversial. During the last decade and a half, there has been a major shift from the use of ephedrine as the recommended obstetric vasopressor to the use of alpha-adrenergic receptor agonists, including metaraminol, ${ }^{1}$ phenylephrine, ${ }^{2}$ and recently norepinephrine. ${ }^{3}$ As I have previously opined, ${ }^{4}$ the debate has now largely shifted from whether we should use alpha agonists to how we should use them. In this issue of the Journal, George et al. ${ }^{5}$ describe the results of a welldesigned randomized double-blinded trial investigating phenylephrine infusions $v s$ phenylephrine boluses in obese parturients and the effect on the incidence of intraoperative nausea and vomiting (IONV). What does this study add to the debate?

First, the study is novel in that the investigators focused specifically on obese parturients. In many previous studies, obesity has been a specified exclusion. Obesity is now a well-recognized worldwide problem for patients and their caregivers; obstetric anesthesiologists are no exception. Obesity poses a number of challenges to obstetric anesthesiologists, including technical challenges, important associated physiological changes, and a high incidence of comorbidities. In the study by George et al., the incidence of hypotension in the infusion group was

W. D. Ngan Kee, BHB, MBChB, MD, FANZCA, FHKCA, FHKAM (Anaesthesiology) ( $\square$ )

Department of Anesthesiology, Sidra Medical and Research Center, P.O. Box 26999, Doha, Qatar

e-mail:wngankee@sidra.org higher than that observed in some studies of non-obese patients. $^{2}$ Although this may relate to differences in infusion regimens, it is interesting to consider whether obesity might predispose to increased hypotension via an effect on the onset speed or extent of the block (although contentious, as highlighted by the authors), aortocaval compression, ${ }^{6}$ or measurement artifacts. ${ }^{7}$ Despite the uncertainty of the explanation, a high predilection to hypotension would put emphasis on recognizing obese parturients as an at-risk population.

Second, this study is important because the authors specified a clinical endpoint, IONV, as the primary outcome. In contrast, it is more difficult to see the clinical significance of outcomes used in the vast majority of previous studies. For instance, the incidences of hypotension or changes in umbilical cord blood gases are often arbitrarily defined. Does it really matter if the blood pressure transiently drops but the mother and fetus are fine, or should it cause concern if there is a 0.05 difference in umbilical arterial $\mathrm{pH}$ ? In the context of older studies, outcomes such as umbilical cord blood gases were important. Before alpha-adrenergic receptor agonists could be considered suitable for routine use, it was necessary to exclude any harmful effects of these drugs on neonatal wellbeing. Now that we are mostly convinced that alphaadrenergic receptor agonists are safe to use in obstetric patients, informed clinicians should look for outcomes that are clinically relevant to their practice. In this respect, IONV stands out because it is both very common and very unpleasant. I suggest that the incidence of IONV should now be regarded as an important clinical indicator of the quality of care in obstetric anesthesia.

What can we learn from the results of the study by George et al.? We could interpret the decrease in the incidence of IONV in the phenylephrine infusion group as 
indicating that this should be the preferred method of administration. However, before reaching this conclusion, it is necessary to conduct a critical examination of the study methodology. Importantly, the bolus group received a phenylephrine bolus as a "rescue" procedure after hypotension had occurred; therefore, it should not be surprising that the incidence of IONV was greater in this group. A more informative design would compare prophylactic infusions with prophylactic boluses. Previously, Doherty et $a .^{8}$ compared the use of phenylephrine infusions vs phenylephrine boluses whenever there was any decrease in blood pressure below baseline. The primary outcome, cardiac output, was not different between groups, but interestingly, there was a smaller decrease in blood pressure below baseline in the first few minutes after spinal injection in patients who received phenylephrine boluses. Similarly, I found that delivery of rapid phenylephrine boluses using a closed-loop computer-controlled delivery system resulted in better control of blood pressure compared with infusions. This was attributed to better matching of rapid boluses to the intermittent nature of noninvasive blood pressure measurements. ${ }^{9}$ Clearly, good control of blood pressure is achievable with intermittent bolus administration.

Conversely, infusions may be advantageous because they might reduce the number of interventions and thus the workload required by physicians. This finding is reported in the current study and has been reported in other studies of phenylephrine infusions. ${ }^{10}$ Bishop et al. ${ }^{11}$ reported that, even in a resource-constrained environment, a prophylactic phenylephrine infusion administered without a syringe pump was an effective method to decrease the incidence of severe hypotension when compared with bolus administration.

Regardless of the method of administration, a take-home message from the results of the study by George et al. is the importance of being proactive with vasopressor administration. Most parturients having spinal anesthesia will experience a decrease in blood pressure; therefore, it makes sense to anticipate this effect and to give a vasopressor in sufficient doses and early enough to minimize the impact of the sympathetic block. The consequences of this approach may be a higher incidence of reactive hypertension ${ }^{2}$ and possibly a transient decrease in cardiac output. ${ }^{12}$ Although the latter effects are not desirable, they have not yet been shown to be associated with measurable harm. In contrast, the alternative, hypotension-associated IONV, is clearly undesirable. Adding a prophylactic antiemetic might further decrease the incidence of IONV. Overall, when comparing the possibility of harm $v s$ benefit, the balance seems weighted towards early proactive administration of vasopressors by whichever route is suited to individual circumstance. This may be even more important in obese patients. This is a small change in practice that can result in a significant benefit for our patients.

\section{La prévention des nausées et vomissements induits par l'hypotension pendant une rachianesthésie lors d'une césarienne chez les parturientes obèses : petite solution à un grand problème?}

De nombreuses études ont été publiées pour examiner le problème de l'hypotension pendant la rachianesthésie lors des césariennes. Toutefois, il n'existe pas de consensus sur sa prise en charge optimale, et bon nombre de chercheurs explorent encore diverses pistes dans ce domaine. Plus particulièrement, le choix et l'utilisation de vasopresseurs sont spécialement controversés. Au cours des quinze dernières années, nous avons assisté à un changement majeur, passant de l'éphédrine comme vasopresseur recommandé pour l'obstétrique à l'utilisation d'agonistes des récepteurs alpha-adrénergiques, notamment le métaraminol, ${ }^{1}$ la phényléphrine ${ }^{2}$ et, plus récemment, la norépinéphrine. $^{3}$ Comme je l'ai précédemment mentionné, ${ }^{4}$ aujourd'hui, le débat ne porte pratiquement plus sur la question de savoir si on devrait utiliser des alpha-agonistes, mais plutôt sur comment les utiliser. Dans ce numéro du Journal, George et coll $^{5}$ décrivent les résultats d'une étude randomisée à double insu bien conçue portant sur des perfusions vs des bolus de phényléphrine chez des parturientes obèses et l'effet de ces deux modalités sur l'incidence des nausées et vomissements peropératoires. Qu'est-ce que cette étude ajoute au débat?

En premier lieu, l'étude est innovante en ce que les chercheurs se sont intéressés spécifiquement aux parturientes obèses. Dans plusieurs études précédentes, l'obésité était un critère d'exclusion spécifié. L'obésité est aujourd'hui un problème mondial bien reconnu, tant pour les patients que pour les personnes qui en prennent soin; les anesthésiologistes obstétricaux ne font pas exception à la règle. L'obésité pose plusieurs défis aux anesthésiologistes obstétricaux, notamment des défis techniques, d'importants changements physiologiques associés, et une incidence élevée de comorbidités. Dans l'étude de George et coll., l'incidence d'hypotension dans le groupe perfusion était plus élevée que celle observée dans certaines études 
s'intéressant à des patientes non obèses. ${ }^{2}$ Bien que cela puisse être lié à des différences dans les taux de perfusion, il est intéressant d'examiner si l'obésité a eu un effet sur le délai d'action ou l'étendue du bloc (bien que cela soit discutable, comme le soulignent les auteurs), sur la compression aorto-cave ${ }^{6}$ et aussi sur les artéfacts de mesure. $^{7}$ Malgré l'incertitude quant à ses causes, une forte prédilection à l'hypotension soulignerait que les parturientes obèses constituent une population à risque.

Deuxièmement, cette étude est importante parce que les auteurs ont spécifié un critère d'évaluation clinique, soit les nausées et vomissements peropératoires, comme critère d'évaluation principal. Par opposition, il est plus difficile de voir la pertinence clinique des critères d'évaluation utilisés dans la grande majorité des études antérieures. Par exemple, l'incidence d'hypotension ou les changements dans les gaz sanguins du cordon ombilical sont souvent définis de manière arbitraire. Est-il vraiment important que la tension artérielle chute provisoirement si la mère et le foetus vont bien? Devrait-on s'inquiéter d'une différence de 0,05 dans le $\mathrm{pH}$ artériel du cordon ombilical? Dans le contexte des études plus anciennes, des critères d'évaluation tels que les gaz sanguins dans le cordon ombilical étaient importants. Avant que les agonistes des récepteurs alpha-adrénergiques ne soient considérés comme adaptés à une utilisation de routine, il était nécessaire d'exclure tout effet néfaste de ces médicaments sur le bien-être du nouveau-né. Maintenant que nous sommes majoritairement convaincus que les agonistes des récepteurs alpha-adrénergiques sont sécuritaires lorsqu'ils sont employés chez les patientes obstétricales, le clinicien informé devrait chercher des critères d'évaluation cliniquement pertinents à sa pratique. Ainsi, les nausées et vomissements peropératoires se distinguent parce qu'ils sont à la fois très fréquents et très désagréables. Je suggère que l'incidence de nausées et vomissements peropératoires soit désormais considérée comme un important indicateur clinique de la qualité des soins en anesthésie obstétricale.

Que pouvons-nous apprendre des résultats de l'étude de George et coll.? Nous pourrions interpréter la diminution de l'incidence de nausées et vomissements peropératoires dans le groupe perfusion de phényléphrine comme indiquant que cette voie d'administration devrait être privilégiée. Toutefois, avant de sauter à cette conclusion, il est nécessaire de réaliser un examen critique de la méthodologie de l'étude. Fait important, le groupe bolus a reçu un bolus de phényléphrine comme intervention « de sauvetage », soit après l'apparition de l'hypotension; par conséquent, il ne devrait pas être surprenant que l'incidence de nausées et vomissements peropératoires soit plus élevée dans ce groupe. Une conception plus instructive aurait comparé des perfusions prophylactiques à des bolus prophylactiques. Par le passé, Doherty et coll. ${ }^{8}$ ont comparé l'utilisation de perfusions de phényléphrine à celle de bolus de phényléphrine à chaque fois qu'il y avait une réduction de la tension artérielle au-dessous des valeurs de base. Aucune différence n'a été observée entre les groupes en matière de débit cardiaque, soit le critère d'évaluation principal, mais, fait intéressant, une réduction moindre de la tension artérielle au-dessous des valeurs de base a été observée au cours des premières minutes suivant l'injection rachidienne chez les patientes ayant reçu des bolus de phényléphrine. De la même manière, dans mon expérience, l'administration de bolus rapides de phényléphrine à l'aide d'un système d'administration géré par ordinateur en circuit fermé a entraîné un meilleur contrôle de la tension artérielle par rapport à une administration par perfusion. Cette amélioration a été attribuée à une meilleure correspondance des bolus rapides à la nature intermittente des mesures automatiques de la tension artérielle. ${ }^{9}$ Clairement, un bon contrôle de la tension artérielle est possible en administrant des bolus de façon intermittente.

À l'inverse, des perfusions pourraient être avantageuses en ce qu'elles pourraient réduire le nombre d'interventions et ainsi la charge de travail des médecins. Cette observation est rapportée dans l'étude en question ici, et a également été mentionnée dans d'autres études portant sur les perfusions de phényléphrine. ${ }^{10}$ Bishop et coll. ${ }^{11}$ ont rapporté que, même dans un environnement où les ressources sont limitées, une perfusion prophylactique de phényléphrine administrée sans pousse-seringue constituait une méthode efficace pour réduire l'incidence d'hypotension grave comparativement à l'administration d'un bolus.

Indépendamment de la méthode d'administration, l'un des messages à retenir des résultats de l'étude de George et coll. est l'importance d'être proactif en ce qui touche à l'administration de vasopresseurs. La tension artérielle diminuera chez la plupart des parturientes recevant une rachianesthésie, c'est pourquoi il est logique d'anticiper cet effet et d'administrer un vasopresseur à une dose suffisante et suffisamment tôt pour minimiser l'impact du bloc sympathique. Les conséquences de cette approche pourraient être une incidence plus élevée d'hypertension réactive $^{2}$ et, possiblement, une réduction temporaire du débit cardiaque. ${ }^{12}$ Bien que ces effets ne soient pas désirables, à ce jour aucune association n'a été démontrée entre leur survenue et des dommages mesurables. L'alternative, en revanche, soit les nausées et vomissements peropératoires associés à l'hypotension, est clairement indésirable. L'ajout d'un antiémétique prophylactique pourrait réduire encore davantage l'incidence de nausées et vomissements peropératoires. Globalement, lorsqu'on compare la possibilité d'un 
dommage $v s$ celle d'un bienfait, il semble que la balance penche vers une administration proactive précoce de vasopresseurs, peu importe le choix de la voie d'administration en fonction des circonstances individuelles. Cela pourrait être encore plus important chez les patientes obèses. Il s'agit d'une petite modification à la pratique qui peut avoir pour effet un avantage significatif pour nos patientes.

Conflicts of interest None declared.

Editorial responsibility This submission was handled by Dr. Philip M. Jones, Associate Editor, Canadian Journal of Anesthesia.

Funding sources Supported by departmental and institutional funding only.

\section{Conflit d'intérêt Aucun.}

Responsabilité éditoriale Cet article a été traité par Dr Philip M. Jones, rédacteur adjoint, Journal canadien d'anesthésie.

Sources de financement Financement départemental et institutionnel seulement.

\section{References}

1. Ngan Kee WD, Lau TK, Khaw KS, Lee BB. Comparison of metaraminol and ephedrine infusions for maintaining arterial pressure during spinal anesthesia for elective cesarean section. Anesthesiology 2001; 95: 307-13.

2. Ngan Kee WD, Khaw KS, Ng FF. Prevention of hypotension during spinal anesthesia for cesarean delivery: an effective technique using combination phenylephrine infusion and crystalloid cohydration. Anesthesiology 2005; 103: 744-50.
3. Ngan Kee WD, Lee SW, Ng FF, Tan PE, Khaw KS. Randomized double-blinded comparison of norepinephrine and phenylephrine for maintenance of blood pressure during spinal anesthesia for cesarean delivery. Anesthesiology 2015; 122: 736-45.

4. Ngan Kee WD. Phenylephrine infusions for maintaining blood pressure during spinal anesthesia for cesarean delivery: finding the shoe that fits. Anesth Analg 2014; 118: 496-8.

5. George RB, McKeen DM, Dominguez, JE, Allen TK, Doyle PA, Habib AS. Randomized trial of phenylephrine infusion versus bolus for nausea and vomiting during cesarean in obese women. Can J Anesth 2018; 65. https://doi.org/10.1007/s12630-0171034-6.

6. Gaiser R. Anesthetic considerations in the obese parturient. Clin Obstet Gynecol 2016; 59: 193-203.

7. Palatini P, Parati G. Blood pressure measurement in very obese patients: a challenging problem. J Hypertens 2011; 29: 425-9.

8. Doherty A, Ohashi Y, Downey K, Carvalho JC. Phenylephrine infusion versus bolus regimens during cesarean delivery under spinal anesthesia: a double-blind randomized clinical trial to assess hemodynamic changes. Anesth Analg 2012; 115: 1343-50.

9. Ngan Kee WD, Tam YH, Khaw KS, Ng FF, Lee SW. Closed-loop feedback computer-controlled phenylephrine for maintenance of blood pressure during spinal anesthesia for cesarean delivery: a randomized trial comparing automated boluses versus infusion. Anesth Analg 2017; 125: 117-23.

10. Siddik-Sayyid SM, Taha SK, Kanazi GE, Aouad MT. A randomized controlled trial of variable rate phenylephrine infusion with rescue phenylephrine boluses versus rescue boluses alone on physician interventions during spinal anesthesia for elective cesarean delivery. Anesth Analg 2014; 118: 611-8.

11. Bishop DG, Cairns C, Grobbelaar M, Rodseth RN. Prophylactic phenylephrine infusions to reduce severe spinal anesthesia hypotension during cesarean delivery in a resource-constrained environment. Anesth Analg 2017; 125: 904-6.

12. Stewart A, Fernando R, McDonald S, Hignett $R$, Jones $T$, Columb $M$. The dose-dependent effects of phenylephrine for elective cesarean delivery under spinal anesthesia. Anesth Analg 2010; 111: $1230-7$. 